\title{
The Robin Hood Index Adjusted for Negatives and Equivalised Incomes
}

\author{
Marion van den Brakel ${ }^{1}$ and Reinder Lok ${ }^{1}$
}

\begin{abstract}
Indisputable figures on income and wealth inequality are indispensable for politics, society and science. Although the Gini coefficient is the most common measure of inequality, the straightforward concept of the Robin Hood index (namely, the income share that has to be transferred from the rich to the poor to make everyone equally well off) makes it a more attractive measure for the general public. In a distribution with many negative values particularly wealth distributions - the Robin Hood index can take on values larger than 1 , indicating an intuitively impossible income transfer of more than $100 \%$. This article proposes a method to normalise the Robin Hood index. In contrast to the original index, the normalised Robin Hood index always takes on values between 0 and 1 and ends up as the original index in a distribution without negatives. As inequality measures are commonly applied to equivalised income, we also introduce a method for adequately transferring equivalised incomes from the rich to the poor within the framework of the (normalised) Robin Hood index. An empirical application shows the effect of normalisation for the Robin Hood index, and compares it to the normalisation of the Gini coefficient from previous research.
\end{abstract}

Key words: Negative wealth; Pietra or Schutz index; normalisation; income inequality; Gini coefficient.

\section{Introduction}

For decades, inequality in income and wealth has been a continuous source of debate in politics, society and science. Studies on income and wealth inequality show a strong correlation with social and socio-economic changes. The OECD $(2014,2015)$ links globalisation and an increasingly flexible labour market to growing inequalities and demonstrates that in some Western countries, increasing inequality has had an inhibitory effect on economic growth. Piketty (2013) stated that growing economic inequality is accompanied by rising mistrust of citizens in fellow citizens and in politics, thus undermining the institutional structures of the society. The potential impact of economic inequality on societal changes, and vice versa, calls for adequate ways to describe the phenomenon.

Various criteria for measuring income (wealth) inequality have been developed over time. However, the complex structure of most of these criteria is a barrier to public

\footnotetext{
${ }^{1}$ Statistics Netherlands, Department of Statistics on Labour, Income and Living conditions CBS-weg 116412 EX Heerlen, the Netherlands. Emails: mhfs@cbs.nl, and rlok@cbs.nl.

Acknowledgments: The authors are grateful to the unknown referees, the associate editors, Ferdy Otten (Statistics Netherlands) and Jan van den Brakel (Statistics Netherlands and Maastricht University) for reading and commenting on earlier drafts of the article. The views in this article are those of the authors and do not necessarily reflect the policy of Statistics Netherlands.
} 
understanding of financial prosperity inequality. Especially national statistical institutes, primarily responsible for providing inequality figures, are challenged with presenting indisputable figures in an appealing manner. The most commonly used measure of inequality is the Gini coefficient, developed in 1912 by the Italian statistician Corrado Gini. The coefficient owes its popularity in particular to its insightful graphic interpretation by the Lorenz curve. Less common, but conceptually much more insightful for the general public is the Robin Hood index, introduced by Gaetano Pietra in 1915 (see Pietra 2014 for an English translation) and also known as the Schutz index (Schutz 1951). The index expresses the share of the total income (or wealth) that has to be transferred from the rich to the poor half, in order to achieve an equal income for each household.

The Gini coefficient and the Robin Hood index normally take on values between 0 and 1 , where 0 stands for perfect equality (everyone has the same income/wealth) and 1 for complete inequality (one household possesses everything). However, in a distribution with negatives, the Gini coefficient may take on values larger than 1, as pointed out by Chen et al. (1982). It is easy to see that the Robin Hood index is also sometimes faced with a distorded and intuitively impossible transfer of more than $100 \%$ of the total income. For instance: to achieve equality, Robin Hood has to transfer EUR 6,000 from a household with an income of EUR 8,000 to its neighbors who suffered losses of EUR 4,000; in other words, 1.5 times the total income (EUR 4,000) has to be shifted.

Elaborating on the work of Chen et al. (1982), Raffinetti et al. (2015) published a method to accurately incorporate negative incomes into the calculation of the Gini coefficient. This article discusses normalisation of the Robin Hood index, using the technique of Raffinetti et al. (2015) to proof the accurateness of the new index. In contrast to the original index, the normalised Robin Hood index always lies between 0 and 1 and ends up as the original index in a distribution without negative values. Distortion is no longer an issue and an outcome range regardless of the income (or wealth) distribution enables a proper inequality comparison between two or more populations, even if negative values occur. Furthermore, an upper and lower limit give meaning to the level of inequality of a distribution. As inequality measures are commonly applied to income and for comparability reasons equivalisation of income is customary international practice (United Nations 2011), we also introduce a method for adequately transferring equivalised incomes from the rich to the poor within the framework of the (normalised) Robin Hood index. An empirical application based on the Income and Wealth Statistics of Statistics Netherlands shows the effect of normalisation for the Robin Hood index as well as the Gini coefficient.

\section{Normalising the Robin Hood Index}

The simple concept of the share of income (or wealth) that has to be transferred from the rich half to the poor in order to achieve equality is captured in the formula of the classical Pietra or Robin Hood index $R$ (see e.g., Pietra 2014; Ricci 1916):

$$
R=\frac{\sum_{i \in D}\left(x_{i}-\mu\right)}{\sum_{i=i}^{N} x_{i}}=\frac{\sum_{i=1}^{N}\left|x_{i}-\mu\right|}{2 \sum_{i=1}^{N} x_{i}}
$$


with $N$ the number of units (households or persons) in the population, $x_{i}$ the income of unit $i, \mu$ the mean income and $D$ the subpopulation of units having an income larger than the mean. In words, $R$ is the ratio of the total of all absolute unit distances to the mean income and twice the total income $T$. In distributions with only nonnegative values the outcome of $R$ is always between 0 and 1 , but as shown from the example in the introductory section this is not necessarily the case if negative values occur.

\subsection{Normalising (Positive Total)}

In a distribution $X=\left(x_{1}, \ldots, x_{N}\right)$ with both positive and negative values and $T$ supposed to be positive, an upper bound of the Robin Hood index is $T^{+} / T$, where $T^{+}$is the total amount of all positive incomes (proposition $A$ ).

Proof of proposition A: using that for any real values $a$ and $b$ the inequality $|a-b| \leq$ $|a|+|b|$ applies and since $\mu>0$, starting from the last term of Equation (1) it is easy to see that

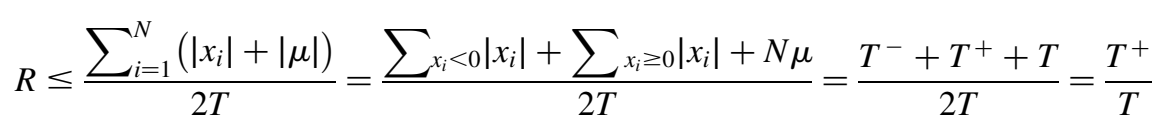

where $T^{-}$is equal to the absolute value of the total amount of all negative incomes.

It now is obvious to define the normalised Robin Hood index $R *$ by dividing $R$ by $T^{+} / T$ :

$$
R^{*}=\frac{\sum_{i \in D}\left(x_{i}-\mu\right)}{T^{+}}
$$

Both the denominator and nominator of $R *$ are not negative by construction, so the lower bound of $R *$ is zero. The upper bound is equal to 1, which directly follows from Equation (2). The thus normalised Robin Hood index $R *$ can be interpreted as the share of positive income that has to be transferred to achieve perfect equality, which intuitively is a logical way for Robin Hood to act. Furthermore, $R^{*}=R$ in case of only nonnegative values.

\subsection{Visual Interpretation}

The normalisation of the Robin Hood index can be interpreted graphically by the Lorenz curve. To illustrate this, first of all note that the classical Robin Hood index (1) of $X$ is identical to the longest vertical distance between the Lorenz curve, which is the cumulative portion of the total income held below a certain income percentile, and the 45-degree line representing perfect equality (see also Pietra (2014), who proved this for distributions with only nonnegative values). Defining this longest distance by $R_{X}$, this proposition $(B)$ can be formulated as: $R=R_{X}$, in a distibution $X$ with positive and negative values and $T>0$.

Proof of propostion B: Let $f$ be the discrete Lorenz curve of $X$ (in which the units are ordered by income), thus $f(k)=\frac{\sum_{i=1}^{k} x_{i}}{T}$. Note that as long as $x_{k}<0$ the Lorenz curve just decreases and gets further away from the equality line (slope $<0$ ). As soon as $x_{k} \geq 0$ the decreasing stops and once $x_{k}>0$ the curve increases (slope $\geq 0$ ). Let $k$ be first unit of $X$ 
for which the slope of the Lorenz curve $f$ is larger than 1. For this slope applies:

$$
\frac{\Delta f}{\Delta i}=\frac{f(k)-f(k-1)}{1 / N}=N\left(\frac{\sum_{i=1}^{k} x_{i}}{T}-\frac{\sum_{i=1}^{k-1} x_{i}}{T}\right)=\frac{N x_{k}}{T}>1,
$$

which implies that $k$ is the first unit for which the income $x_{k}$ is larger than the mean $\mu$. From unit $k$ on, the vertical distance between the Lorenz curve and the equality line (with constant slope 1) will only become smaller (as long as the slope of the Lorenz curve is smaller than 1 , the distance between the curve and the equality line grows). The maximum vertical distance is therefore found at $m=k-1$ and is equal to

$$
\frac{m}{N}-\frac{\sum_{i=1}^{\mathrm{m}} x_{i}}{T}=\frac{m T}{N T}-\frac{\sum_{i=1}^{\mathrm{m}} x_{i}}{T}=\frac{m \mu}{T}-\frac{\sum_{i=1}^{m} x_{i}}{T}=\frac{\sum_{i \in D^{*}}\left(\mu-x_{i}\right)}{T}
$$

with $D *$ the subpopulation of units having an income smaller than or equal to the mean. Note that Equation (5) is equal to the classical Robin Hood index (1) and for the proof it only matters that $T$ is positive.

Distribution $X$ has positive as well as negative values and the highest value of the Lorenz curve is equal to 1 and the lowest to $\frac{-T^{-}}{T}$. The difference between the highest and lowest value $(d)$ is obviously an upper bound for $R_{X}$. As $d$ equals $1+\frac{T^{-}}{T}=\frac{T^{+}}{T}$, which is just the ratio derived in Equation (2), the nomalised Robin Hood index can be interpreted as the ratio between distance $R_{X}$ and distance $d$.

Example To further clarify the visual interpretation consider the distribution with values $(-8 ;-3 ; 3 ; 8 ; 10)$ and its Lorenz curve in Figure 1 . For this distribution the distance $R_{X}$ is

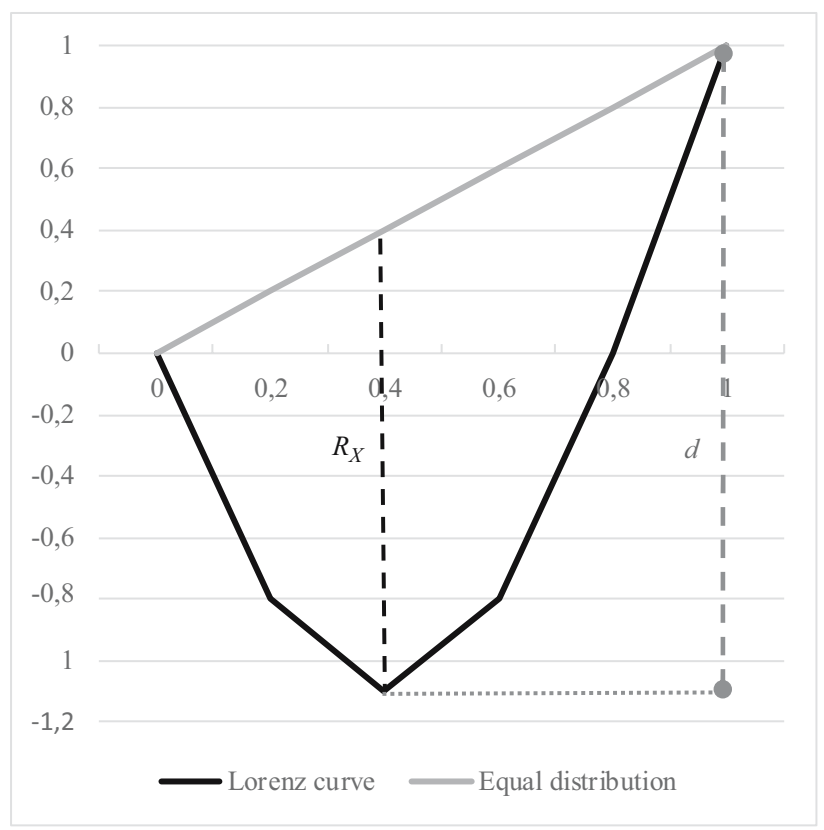

Fig. 1. Distribution $(-8,-3,3,8,10)$. 
equal to 1.5. The distance between the highest and lowest value of the Lorenz curve equals 2.1. Dividing 1.5 by 2.1 gives a value of 0.71 . Exactly the same value can be achieved using Equation (3).

\subsection{No Over-Normalisation}

The normalisation of the Robin Hood index could be too rough in the way that ratio $T^{+} / T$ might be too large. To prove that no over-normalisation is done by dividing Equation (1) by this ratio (proposition $C$ ), consider the corresponding distribution with maximum inequality $Z=\left(-T^{-}, 0, \ldots, 0, T^{+}\right)$used by Raffinetti et al. (2015) to normalise the Gini coefficient.

Proof of proposition $C$ : Not only in terms of the Gini coefficient, but also in terms of the classical Robin Hood index, $Z$ matches to maximum inequality. After all, the longest vertical distance from the Lorenz curve of $Z$ to the equality line is previously proven to be identical to the classical Robin Hood index of $Z$ and from Equation (1) equals $\frac{T^{+}-\mu}{T}=\frac{T^{+}}{T}-\frac{1}{N}$. As $N \rightarrow \infty$ this approaches $\frac{T^{+}}{T}$, which is already shown to be larger than the classical Robin Hood index of $X$.

For distribution $Z$ the normalised Robin Hood index is equal to $R_{Z}^{*}=$ $\frac{T^{+}-\frac{\left(T^{+}-T^{-}\right)}{N}}{T^{+}}=1-\frac{1}{N} \frac{T}{T^{+}}$. Obviously as $N \rightarrow \infty, R_{Z}^{*}$ will approximate 1 , which means that over-normalisation is not the case.

\subsection{Normalising (Zero or Negative Total)}

Up to now the total income $T$ of distribution $X=\left(x_{1}, \ldots, x_{N}\right)$ was assumed to be positive. For the normalised Robin Hood index, however, it is no problem if $T$ is zero, that is $T^{-}=T^{+}(>0)$. In this (rare) case $R^{*}$ equals 1 . In the special case where all values are zero, $R$ and $R *$ are undefined, but since this refers to an equal distribution, the (normalised) Robin Hood index can be defined as 0 . When $T$ is negative $\left(T^{-}>T^{+}\right)$the normalised Robin Hood as formulated in Equation (3) cannot be applied, because to deduce it, the total was assumed to be positive. A solution for this problem can be found in the mirrored distribution of $X$. For this, first notice that the maximum vertical distance of the Lorenz curve of $X$ to the equality line is equal to that of the Lorenz curve of $Y=-X$ (proposition $D$ ).

Proof of propostion D: For every $l \leq N$ the value of the Lorenz curve $f$ of $X$ is at least $\frac{l}{N}$, meaning that the Lorenz curve $f$ lies above or on the equality line. To see this, let $k<N$ be the last unit in the (ranked) distribution $X$ for which $x_{k} \leq \mu$. Then, since $T$ and $\mu$ are equal to the negation of the total $T_{Y}$ and the mean $\mu_{Y}$ of distribution $Y$ respectively, for every $l \leq k$

$$
y_{l} \geq \mu_{Y} \text { and } f(l)=\frac{\sum_{i=1}^{l} x_{i}}{T}=\frac{\sum_{i=1}^{l}\left(-y_{i}\right)}{-T_{Y}} \geq \frac{l \mu_{Y}}{N \mu_{Y}}=\frac{l}{N}
$$

and for every $l>k$

$$
f(l)-\frac{l}{N}=\frac{\sum_{i=1}^{l} x_{i}}{T}-\frac{l}{N}>\frac{\sum_{i=1}^{l} \mu}{T}-\frac{l}{N}=0 .
$$

Opposite to the situation in which the Lorenz curve lies beneath the equality line $(T>0)$, the maximum vertical distance of $f$ to the equality line is found at the point from where on 
the slope of $f$ only takes on values smaller than 1 . For the slope at this point, say at unit $k$, analogous to Equation (4) applies $\frac{\Delta f}{\Delta i}=\frac{N x_{k}}{T}<1$ which comes down to $y_{k}<\mu_{Y}$. This exactly corresponds to the unit at which the vertical distance of the Lorenz curve of $Y$ to the equality line is at the largest, as seen before in Equation (5).

This means that the normalised Robin Hood of $X$ (with negative total) can logically be defined as the normalisation of that of $Y=-X$. Normalising the Robin Hood index of $Y$ means dividing it by $\frac{T_{Y}^{+}}{T_{Y}}$ and since $T_{Y}^{+}=\sum_{i=1}^{N} \max \left(0, y_{i}\right)=\sum_{i=1}^{N} \max \left(0,-x_{i}\right)=$ $\left|\sum_{i=1}^{N} \min \left(0, x_{i}\right)\right|=T^{-}$the normalised index for distribution $X$ is where $T^{-}>T^{+}$equals

$$
R_{Y}^{*}=\frac{\sum_{i \in D_{Y}}\left(y_{i}-\mu_{y}\right)}{T_{Y}^{+}}=\frac{\sum_{i \in D_{Y}}\left(-x_{i}+\mu\right)}{T^{-}}=\frac{\sum_{i \in D^{*}}\left(\mu-x_{i}\right)}{T^{-}}=\frac{\sum_{i \in D}\left(x_{i}-\mu\right)}{T^{-}}
$$

Combining Equations (3) and (6) the definition of the normalised Robin Hood index for a non-zero distribution $X$ is:

$$
\frac{\sum_{i \in D}\left(x_{i}-\mu\right)}{\max \left(T^{+}, T^{-}\right)}
$$

with mean $\mu$ and $T^{+}, T^{-}$and $D$ as aforementioned.

Example In the distribution $X=(-8 ;-3 ; 0 ; 3)$ with positive as well as negative values the sum of the absolute negatives exceeds the sum of the positives. Applying Equation (3) would return a normalised Robin Hood index of 7/3. The longest vertical distance $R_{X}$ from the Lorenz curve of $X$ to the equality line is the same as that of the curve mirrored in the equality line (see Figure 2). This mirrored Lorenz curve belongs to the distribution $Y=$

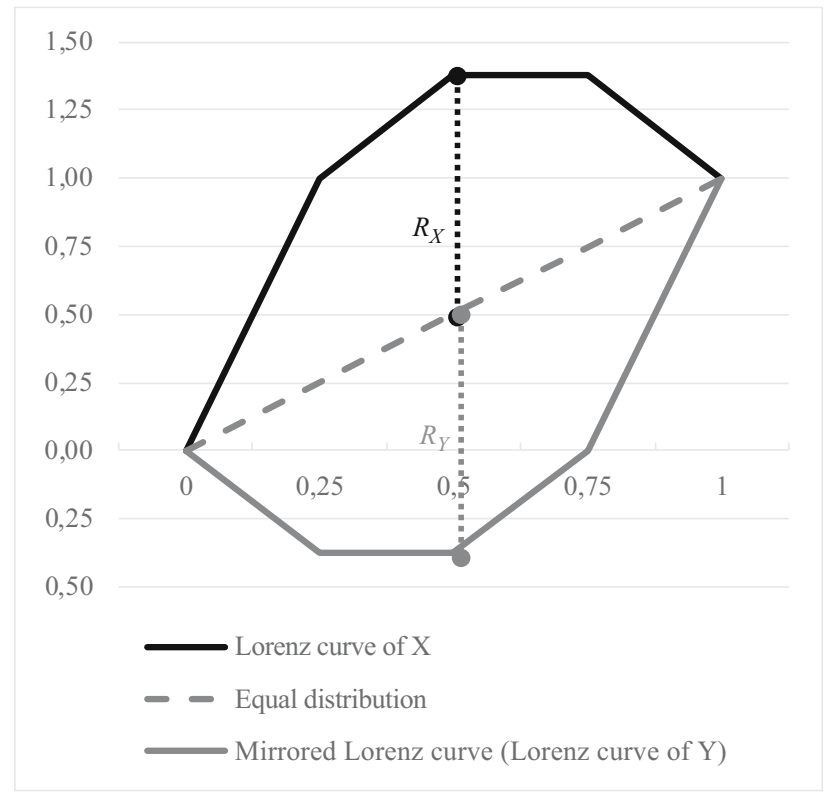

Fig. 2. Distribution (-8, -3, 0, 3). 
$(-3 ; 0 ; 3 ; 8)$, that is, to the distribution $-X$ in which the total of the positive values exceeds the total of the absolute negative incomes. The normalised Robin Hood $R_{Y}^{*}$ derived from Equation (3) is 7/11, which is exactly the same value achieved from Equation (7).

In the special case where all incomes of a distribution $X=\left(x_{1}, \ldots, x_{N}\right)$ are negative, the income inequality can be calculated by the mirrored distribution $Y=\left(y_{1}, \ldots, y_{N}\right)=$ $\left(\left|x_{1}\right|, \ldots,\left|x_{N}\right|\right)$ and using the classical Robin Hood index (1).

\subsection{Properties of the Normalised Robin Hood Index}

For a distribution $X=\left(x_{1}, \ldots, x_{N}\right)$ with positive as well as negative values (i.e., $T^{+}>0$ and $\left.T^{-}>0\right)$ a scale 'invariance' property can be formulated for the normalised Robin Hood index:

If $\left(T^{+} \neq T^{-}\right)$then for every constant $\alpha \neq 0$ the normalised Robin Hood index of $Y=\alpha X$ is equal to $\frac{\sum_{i \in D}\left(x_{i}-\mu\right)}{\max \left(T^{+}, T^{-}\right)}$.

Other features the normalised Robin Hood index (just like the classical index) meets are for instance symmetry (swapping the income of two households leaves the index unchanged) and population size independency (merging two or more identical distributions does not influence the outcome of the index). The Pigou-Dalton criterion only holds for incomes shifted from the rich (individuals having an income above the mean) to the poor (income below the mean), and vice versa. Incomes transferred within the rich (poor) are not signaled by the (normalised) Robin Hood index. The normalised Robin Hood index satisfies boundedness, such that for every distribution the same upper and lower limit apply. This makes comparison of subpopulations possible and gives meaning to the level of inequailty. The traditional Robin Hood index meets the property of boundedness in distributions with solely nonnegatives. Decomposition of an inequality measure is a desirable but not necessary feature. Habib (2012) developed a method to decompose the traditional Robin Hood index. This method can easily be applied to the normalised Robin Hood index as well.

\section{Transferring Equivalised Incomes}

Multi-person households mostly have more income than a single person. However, it matters a lot how many people within a household have to live on a certain income. It therefore makes no sense to determine income inequality without taking into account the size of the household. To make households of different sizes and composition comparable, incomes are equivalised.

Income is equivalised by dividing the household income by a factor that expresses the economies of scale when running a joint household. Single-person households have been chosen as the standard. The factor is set to 1 for these households. For multi-person households the factor depends on the equivalence scale that is chosen. Various alternative equivalence scales have been developed worldwide. International scales that are frequently used within the OECD countries are the modified equivalence scale and the square root scale (OECD 2013). Using the square root scale, a single person with a disposable income of EUR 10,000 and a couple with a disposable income of EUR 14,100 
are at the same level of prosperity: after equivalisation, the purchasing power for both households is EUR 10,000.

With equivalised incomes, Robin Hood's job is a bit more complex. The transfer of income from rich to poor should be done in such a way that afterwards every household has the same equivalised income and the total unequivalised income of the population remains the same. For clarification, consider the following situation.

Example A couple $A$ has a disposable income of EUR 1,500, their single-person neighbor $B$ has no income at all. Assuming for the sake of simplicity an equivalence factor of 1.5 for couples, the average equivalised income is EUR 500. If Robin Hood were to transfer equivalised incomes, couple $A$ would have to hand over EUR 500 to individual $B$. This is half the total equivalised income, which implies the Robin Hood index $R=1 / 2$. Counting back this means that couple $A$ has EUR 750 to spend and individual $B$ EUR 500 . Has Robin Hood put EUR 250 in his own pocket?

By shifting not with 'fictional' (equivalised) but with genuine money, Robin Hood can prevent defamation. Because the couple $A$ shares a household, they do not count for 2 but for 1.5. The total disposable income of EUR 1,500 must therefore be distributed in such a way that couple $A$ has 1.5 times as much as individual $B$. Robin Hood calculates that $A$ has to hand over EUR 600 to $B$, after which the couple has EUR 900 to spend. This means that $2 / 5$ of the total income has been transferred in order to get equal equivalised incomes.

In general, this means that the total of non-equivalised incomes must be evenly distributed over a population of size equal to the sum of the equivalence factors. Based on the traditional Robin Hood index, the proper transfer of equivalised incomes $X=$ $\left(x_{1}, \ldots, x_{N}\right)$ is expressed by:

$$
R_{\text {equi }}=\frac{\sum_{i \in B}\left(x_{i}-\vartheta\right) e_{i}}{\sum_{i=1}^{N} y_{i}}
$$

where $y_{i}$ is the unequivalised income of household $i, e_{i}$ the equivalence factor, $\vartheta$ the mean of all unequivalised incomes of the population of size $M=\sum_{i=1}^{N} e_{i}$ :

$$
\vartheta=\frac{\sum_{i}^{N} y_{i}}{M},
$$

and $B$ the subpopulation of households with $y_{i}>\vartheta$. It is straightforward to derive an expression for $R_{\text {equi }}$ in case of negative (and positive) incomes and the total $T_{Y}=\sum_{i=1}^{N} y_{i}>0$ :

$$
R_{\text {equi }}^{*}=\frac{\sum_{i \in B}\left(x_{i}-\vartheta\right) e_{i}}{T_{Y}^{+}}
$$

whit $T_{Y}^{+}$the sum of all positive unequivalised incomes. If the total is negative, Equation (7) can be applied. 


\section{An Application}

In this section, the impact and relevance of normalising the Robin Hood index is shown by giving some examples of the Income and Wealth Statistics (IWS) of the Netherlands. Furthermore, a comparison with the Gini coefficient is made here. Income inequality is hardy affected by the normalisation of the Robin Hood index or the Gini coefficient. Over the years, the normalised figures for equivalised disposable incomes (see CBS Statline 2020a, where normalisation in accordance with Equation (8) and Raffinetti et al. (2015) respectively is applied) were slightly smaller than the not-normalised income inequalities. For instance, the difference in 2017 was less than $0.2 \%$. Normalisation has more impact on wealth inequality. This is because the wealth of almost $20 \%$ of the households in 2017 is negative: their liabilities (mortgage debts and consumer credit) transcend their assets (mainly bank balances, shares, real estate, and business capital). A negative income is much less common ( $0.5 \%$ in 2017). For both inequality measures, the impact of normalisation grows until 2014, after which it decreases (see Figure 3). This had to do with the economic climate in this period. As a result of the economic crisis that started at the end of 2008, more and more households were faced with negative wealth, especially due to falling house prices. From 2014, the Dutch economy recovered, house prices rose and the number of households with negative wealth decreased again. Note that compared to the Robin Hood index, normalising wealth inequality with the Gini coefficient has more effect. Normalisation reduced the wealth inequality of the Robin Hood index by $4 \%$ in 2017 . Using the Gini coefficient, this figure was twice as large (7.9\%). This is simply because the factor used to normalise the Gini coefficient, that is, $\left(T^{+}+T^{-}\right) / T$ in large populations (see Raffinetti et al. 2015), is larger than that of the Robin Hood index derived in Equation (2).

For certain groups of households in which relatively many negative wealth values occur, like households having a young main earner or households in which the main earner

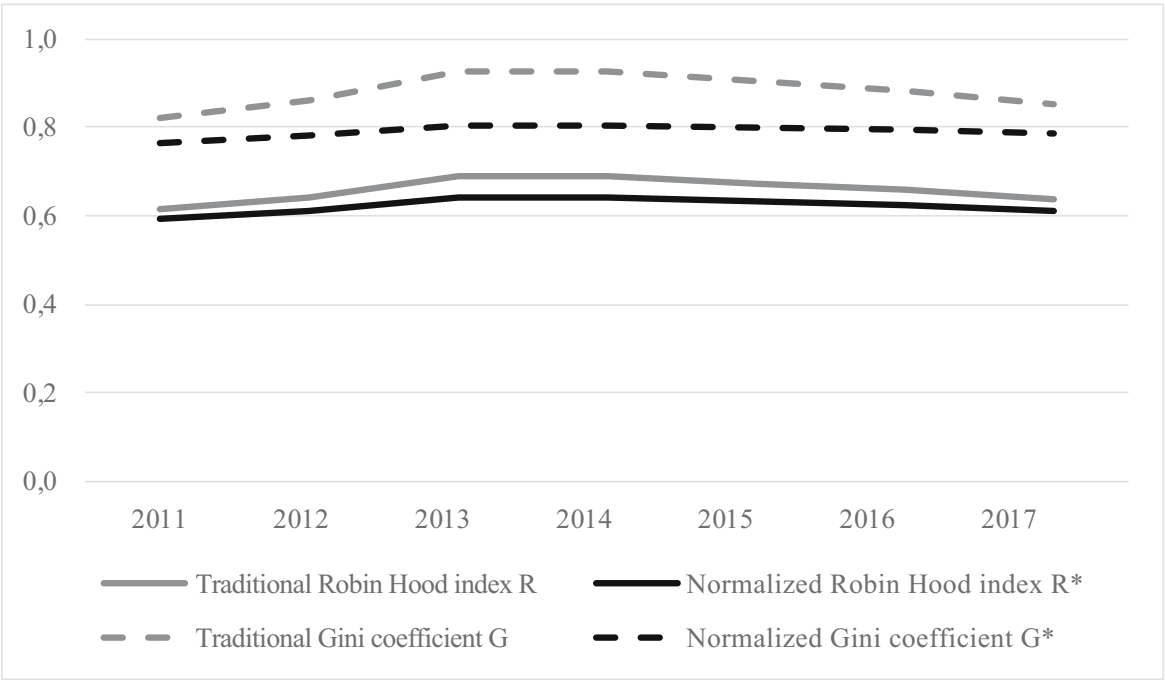

Fig. 3. Wealth inequality of households in the Netherlands.

1) $G *$ in accordance with Raffinetti et al. 2015.

2) Descriptive statistics can be found on CBS StatLine (2020b). 
has a non-Western migration background, the traditional Robin Hood index and the Gini coefficient take on values larger than 1 (see Table 1). Normalisation provides wealth inequality values smaller than 1 . Note that normalisation enables to compare inequalities of different populations. Young households (main earner younger than 25 years) seem to have a much higher ineqality than the group with a non-Western migration background, according to standard inequality scales. However, after normalisation they appear to be quite comparable. The many negative wealth cases (mostly student loans in the young group) did indeed bias the inequality scale to a non-interpretable level.

Table 1. Wealth Households by Characteristics of Main Earner 2017.

\begin{tabular}{|c|c|c|c|c|c|}
\hline & $\begin{array}{l}\text { Households with } \\
\text { negative wealth (\%) }\end{array}$ & $\mathrm{R}$ & $\mathrm{R}^{*}$ & G & $\mathrm{G}^{*}$ \\
\hline \multicolumn{6}{|l|}{ Age } \\
\hline younger than 25 & 42 & 1,353 & 0,810 & 2,039 & 0,870 \\
\hline 25 to 45 & 32 & 0,855 & 0,710 & 1,188 & 0,844 \\
\hline 45 to 65 & 16 & 0,593 & 0,572 & 0,811 & 0,757 \\
\hline 65 and over & 4 & 0,516 & 0,513 & 0,710 & 0,700 \\
\hline \multicolumn{6}{|c|}{ Migration background } \\
\hline Dutch & 17 & 0,603 & 0,581 & 0,817 & 0,761 \\
\hline Western & 20 & 0,708 & 0,675 & 0,920 & 0,838 \\
\hline non-Western & 32 & 1,032 & 0,808 & 1,403 & 0,902 \\
\hline
\end{tabular}

1) Traditional (G) and normalised Gini coefficient $G^{*}$ in accordance with Raffinetti et al. 2015.

2) Descriptive statistics can be found on CBS StatLine (2020b).

\section{Conclusion and Discussion}

This article proposed a method to normalise the Robin Hood index in order to deal with negative values in an income or wealth distribution. The normalised index expresses the share of the total positive amount of income or wealth (instead of the total amount, as in the traditional Robin Hood index) that has to be transferred from the rich half to the poor half in order to achieve perfect equality. The method provides an expression for normalisation, even in extreme distributions with zero or negative total. A proper (normalised) way to shift equivalised incomes from the rich to the poor is also incorporated. An application to the prosperity distributions of households in the Netherlands shows that normalisation is especially necessary for wealth inequality, since households with negative wealth are much more common than households with negative income. The development over time of income and wealth inequality after normalisation appears to be the same as before.

Although the Gini coefficient is widely used, its concept is more abstract than that of the Robin Hood index. The simple perception of the Robin Hood index makes it an accessible and understandable measure of income (or wealth) inequality. Moreover, in contrast with similarly easy measures that compare the top and bottom of a distribution (like the 80/20 ratio) or the share of the rich in the total wealth (see e.g., Piketty 2014), the Robin Hood index takes into account each individual value of the population. Another disadvantage of measures such as the 80/20 ratio is their inability to cope with negative values. This 
underlines the significance of the normalised Robin Hood as a measure for inequality to serve a broad public, certainly since it meets several desirable features including symmetry, population size independency and decomposability. As the Pigou-Dalton criterion is only partly met, additional analyses of inequality using the (normalised) Gini coefficient are recommended.

In accordance with international standards, the disposable income does not include quaternary income components. Specifically, this means that both the social benefits received in kind (such as free education and medical care) and the benefits of collective goods (infrastructure and the like) are not taken into account in the disposable income. Therefore caution is required when comparing income inequality in the Netherlands with that in other countries. If the quaternary benefits are at a lower level elsewhere, the observed differences can quickly lead to distorted insights.

The ways in which wealth is measured internationally may differ even more (Balestra and Tonki 2018). In the Netherlands, for instance, when determining the mortgage debt, any accumulated assets with regard to savings and investment mortgages have not been included because the underlying data are lacking. Whether or not pension entitlements and other social security entitlements are counted as assets may also differ among countries, as well as equivalisation of wealth. The figures on income and wealth inequality are therefore primarily intended to monitor developments within a country, especially if normalisation is applied.

\section{References}

Balestra, C., and R. Tonki. 2018. Inequalities in household wealth across OECD countries: Evidence from the OECD Wealth Distribution Database. OECD Statistics Working Paper Series. Available at: www.oecd.org. (accessed September 2019).

CBS StatLine. 2020a. Inequlity of income and wealth (in Dutch). Available at: http://opendata.cbs.nl/statline/\#/CBS/nl/dataset/84476NED/table?dl = 49EF5 (accessed January 2021).

CBS StatLine. 2020b. Welfare of households; key figures. Available at: http://opendata. cbs.nl/statline/\#/CBS/en/dataset/83739ENG/table?dl = 49EF4 (accessed January 2021).

Chen, C.-N., T.-W. Tsaur, and T.-S. Rhai. 1982. “The Gini coefficient and negative income." Oxford Economic Papers 34 (3): 473-478. http://dx.doi.org/10.1093/ oxfordjournals.oep.a041563.

Habib, E.A. 2012. "On the decomposition of the Schutz coefficient; an exact approach with an application." Electronic Journal of Applied Statistical Analysis 5(2) : 187-198. DOI: http://dx.doi.org/10.1285/i20705948v5n2p187.

OECD. 2013. OECD Framework for statistics on the distribution of household income, consumption and wealth. Available at: www.oecd.org (accessed September 2019).

OECD. 2014. Focus on Inequality and Growth. Paris: OECD Publishing.

OECD. 2015. In It Together: Why Less Inequality Benefits All. Paris: OECD Publishing. Pietra, G. 1915. "Delle relazioni tra indici di variabilità." Atti del reale Istituto Veneto di Scienze, Lettere ed Arti 74(2). 
Pietra, G. 2014. "On the relations between variability indices (Note I)." METRON 72: 5-16. DOI: https://doi. org/10.1007/s40300-014-0034-3.

Piketty, T. 2014. Capital in the Twenty-First Century. Harvard University Press.

Raffinetti, E., E. Siletti, and A. Vernizzi. 2015. "On the Gini coefficient normalization when attributes with negative values are considered." Stat Methods Appl 24: 507-512. DOI: http://dx.doi.org/10.1007/s10260-014-0293-4.

Ricci, U. 1916. "L'indice di variabilità e la curva dei redditi." Giornale degli Economisti e Rivista di Statistica LIII: 177-228. serie terza, anno XXVII. Available at: https://www. jstor.org/stable/23225478 (accessed October 2021).

Schutz, R. 1951. "On the measurement of income inequality." The American Economic Review 41: 107-122. Available at: On the Measurement of Income Inequality (jstor.org) (accessed April 2021).

United Nations. 2011. Canberra Group, Handbook on Household Income Statistics (second edition). New York and Geneva. Available at: Canbera_Handbook_2011_WEB.pdf (unece.org) (accessed April 2021).

Received October 2019

Revised May 2020

Accepted April 2021 